\title{
Region of Interest Prediction using Segmentation
}

\section{M. Merlin Livingston, S. Mary Cynthia}

\begin{abstract}
Segmentation separates an image into different sections badsed on the desire of the user. Segmentation will be carried out in an image, until the region of interest (ROI) of an object is extracted. Segmentation reliability predicts the progress of the various segmentation techniques. In this paper, various segmentation methods are proposed and quality of segmentation is verified by using quality metrics like Mean Squared Error (MSE),Signal to Noise Ratio (SNR), Peak- Signal to Noise Ratio (PSNR), Edge Preservation Index (EPI) and Structural Similarity Index Metric (SSIM).

Keywords: Segmentation, ROI, EPI and SSIM
\end{abstract}

\section{INTRODUCTION}

In the modern world, there are many new technologies are emerging in the field of image processing, especially in image segmentation. In this paper, important segmentation methods like color, texture, morphological operation based segmentation, power-law transformation based segmentation and watershed based segmentation are described. The efficiency of the image will be measured with different metrics. PSNR: The PSNR value must be high to produce high quality image. SSIM : Comparison between two images can be predicted by SSIM. EPI: The EPI value must be high to maintain edges.

\section{RELATED WORK}

Segmentation is a process of representing image in an effective manner to analyze. The region of interest is determined using segmentation. The result of image segmentation is a set of patterns received from the cluster of image. The segmentation will be carried out based on the aspects like color, intensity, or texture. Color-based methods are based on the perception of the homogenous and non homogeneous property of the color of the image. Clustering method, extract homogeneous color regions based mostly on color similarity of the image. The desired regions are identified using spatial information and geometrical constraints as specified in Pillai.A.V., et. al.(2013)[1]. Hill Climbing algorithm provides the parameters for deciding on the number of clusters[2]. Hase.H., et.al.(2001)[3],developed a connected component primarily based technique for color scripts. The image is separated into many binary images depends on the histogram analysis. Character string in an image will be identified based on mean and standard deviation. Kim.K.I., et. al.(2003)[4],grasp that SVM features competently depends on kernel functions. A Continuously Adaptive Mean Shift algorithm(CAMSHIFT) is implemented for localization of desired image.

Revised Manuscript Received on June 10, 2020.

* Correspondence Author

L.M. Merlin Livingston*, Jeppiaar Institute of Technology, Sriperumbudur, Tamil Nadu, India. E-mail: merlinlivingston@yahoo.com

S. Mary Cynthia, Jeppiaar Institute of Technology, Sriperumbudur, Tamil Nadu, India. E-mail: flower.jacily@gmail.com

(c) The Authors. Published by Blue Eyes Intelligence Engineering and Sciences Publication (BEIESP). This is an open access article under the CC BY-NC-ND license (http://creativecommons.org/licenses/by-nc-nd/4.0/)
This paper describes various segmentation techniques, such as Color based Segmentation, Texture based Segmentation, Morphological based Segmentation, Power-Law transformation based segmentation and Watershed based segmentation.

\section{IMPLEMENTATION}

Selections of proper segmentation methods are very important to get accurate output. The quality of the segmentation is determined from the calculation of quality metrics.

\subsection{Colour Based Segmentation}

For colour based segmentation [5,6] the image is segmented based on the colours present in the image. Clavelli et al. [7] considered the color information and spatial distribution of characters for segmentations. Components are connected based on Red Green Blue (RGB) color similarities in adjacent pixels.

For example consider the input image given in figure 1.

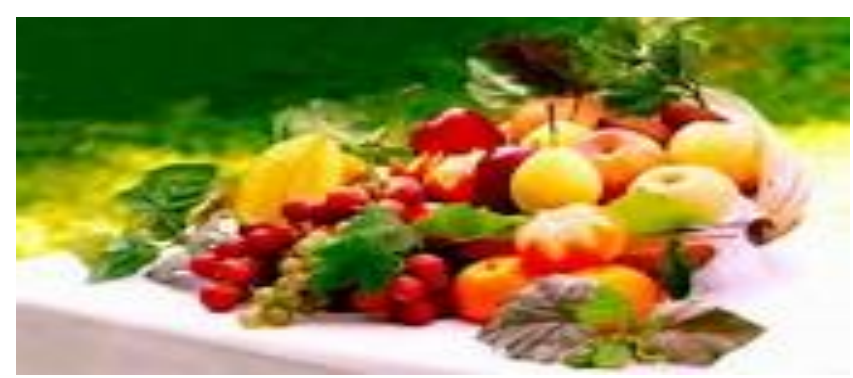

Figure 1. Input image for colour based segmentation.

The above image consists of three major colours. The colour based segmentation splits the regions with different colours separately. The output image is given in figure 2 .

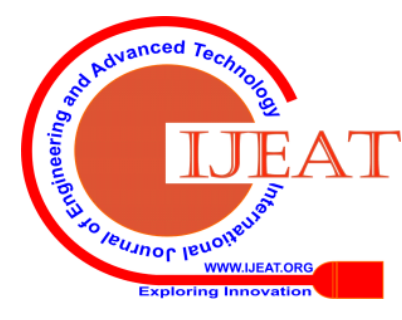




\section{Region of Interest Prediction using Segmentation}
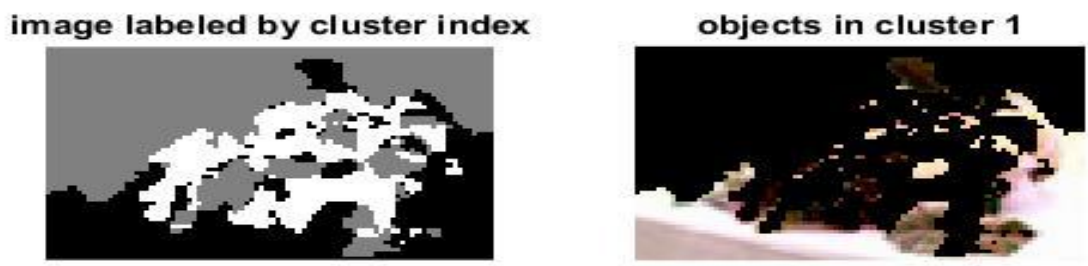

objects in cluster 2

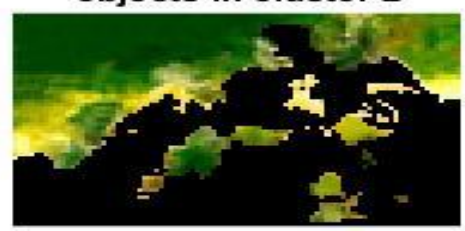

objects in cluster 3

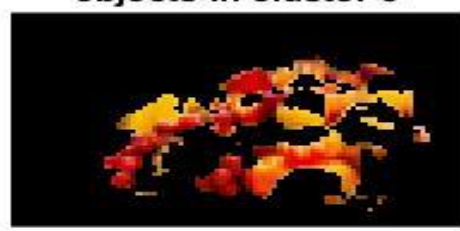

Figure 2. Output image for colour based segmentation

The quality metrics for the above outputs are given in Table 1.

Table 1. Quality metrics for colour based segmentation

\begin{tabular}{|c|c|c|c|}
\hline $\begin{array}{c}\text { Quality } \\
\text { Metrics }\end{array}$ & Cluster 1 & Cluster 2 & Cluster 3 \\
\hline MSE & 669.0342 & 659.1766 & 705.4010 \\
\hline SNR & 19.9080 & 19.4554 & 19.6062 \\
\hline PSNR & 20.4635 & 20.0812 & 20.2624 \\
\hline EPI & 0.6435 & 0.6737 & 0.6668 \\
\hline SSIM & 0.8950 & 0.8983 & 0.8903 \\
\hline
\end{tabular}

\subsection{Texture Based Segmentation}

In texture based segmentation method the image is segmented based on the textures present in the image. The Wavelet transform is used for Texture based segmentation.Wavelet transform uses a pair of high and low pass filters to molder the image into orientation and wavelet coefficients called sub bands. Dyadic decomposition can be used to identify textures effectively. Textured image is
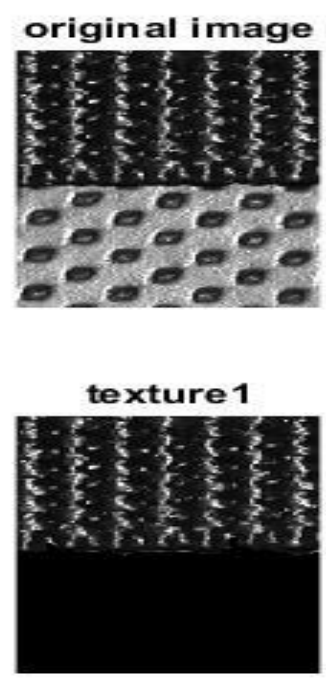

classified into regions to determine homogeneous textured region.For example consider the image shown in figure 3.

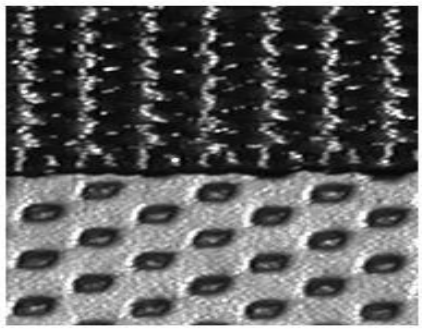

Figure 3. Input image for texture based segmentation

In the above image, two textures are present, one in the upper portion and the second one in the lower portion. The texture based segmentation extract the two textures separately as shown in figure 4.
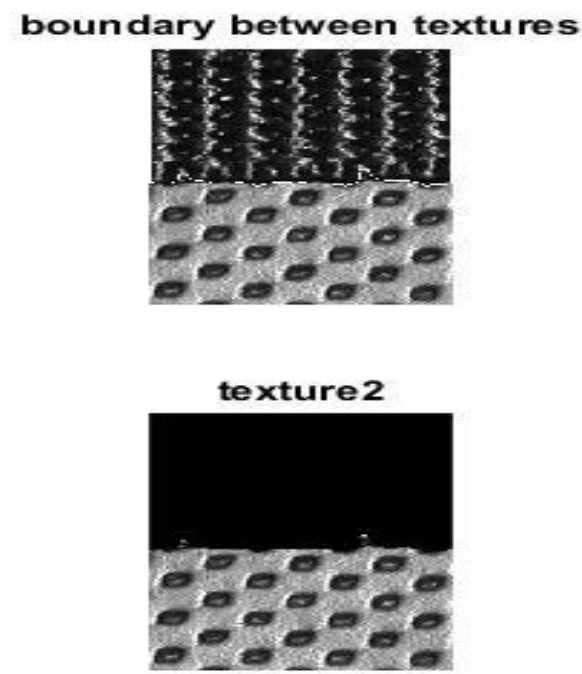

Figure 4. Output image for texture based segmentation

The quality metrics for the above outputs are given in Table 2

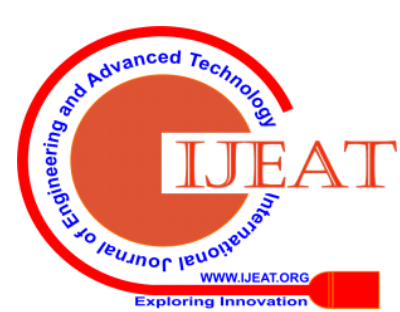


Table 2. Quality metrics for texture based segmentation

\begin{tabular}{|c|c|}
\hline MSE & 574.2320 \\
\hline SNR & 19.1890 \\
\hline PSNR & 20.7466 \\
\hline EPI & 0.6725 \\
\hline SSIM & 0.8405 \\
\hline
\end{tabular}

\subsection{Morphological Operation Based Segmentation}

Morphological operation based segmention [8] is mainly based on two operations, first one dilation and second one erosion. Morphological technique process the images primarily based on shapes. In morphological operation, output image depends on structuring element. By selecting the range and pattern of the neighborhood, make a morphological function that is prone to precise patterns in the input image.

Dilation and erosion are the significant morphological operations. In dilation,object boundaries in an image are increased by adding pixels. In erosion, object boundaries in an image are decreased by removing pixels.The addition and removal of pixels in an image are determined by the size and frame of the structuring element.

According to Vaddi.R.S.,et. al.(2011) [9]the boundaries of each region can be determined by subtracting the eroded image from the initial image.

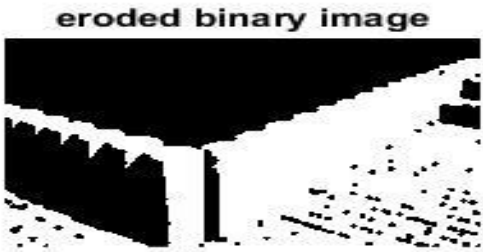

In text information extraction system, the resulting image consists of text regions and small nontext regions in the form of binary.To remove the small nontext regions, morphological operation is used. The dilation operator is applied in the system to connect the text regions and mark the boundary boxes around the text region.

Consider the following input image given in figure 5 .

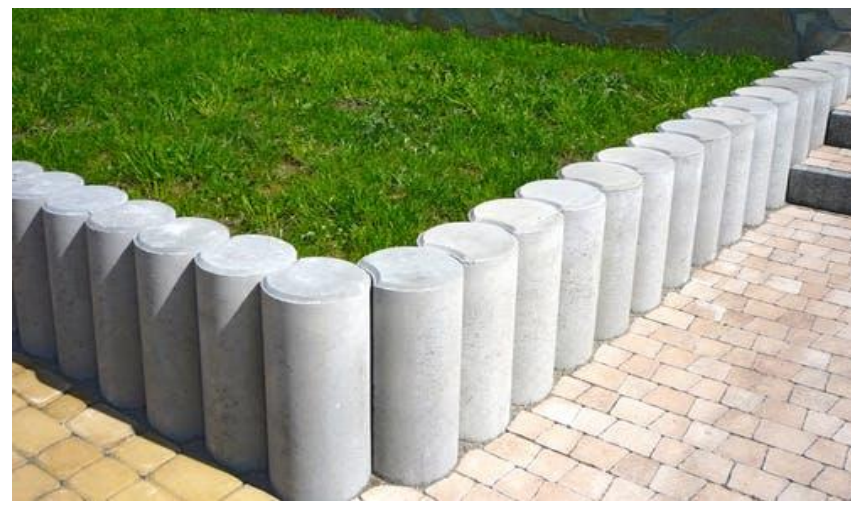

Figure 5. Input image for morphological operation based segmentation

The morphological based segmentation output is shown in figure 6 .

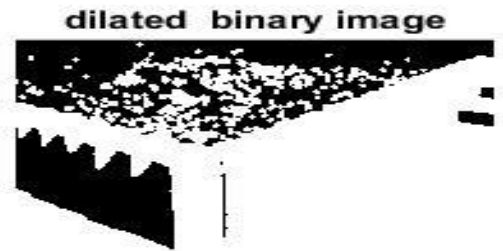

Figure 6. Output image for morphological operation based segmentation Table 3

The quality metrics for the above outputs are given in

Table 3. Quality metrics for morphological operation based segmentation

\begin{tabular}{|c|c|c|}
\hline Quality Metrics & Eroded output & Dilated output \\
\hline MSE & 623.5608 & 646.9517 \\
\hline SNR & 18.4358 & 19.1840 \\
\hline PSNR & 20.1187 & 20.2483 \\
\hline EPI & 0.7674 & 0.7940 \\
\hline SSIM & 0.8905 & 0.8804 \\
\hline
\end{tabular}

\subsection{Power-Law Transformation Based Segmentation}

In power-law transformation based method the segmentation is based on power-law that is variation in one quantity is in the power of variation in another quantity. Power-law transformation based segmentation is applied to the input given in figure 7 .

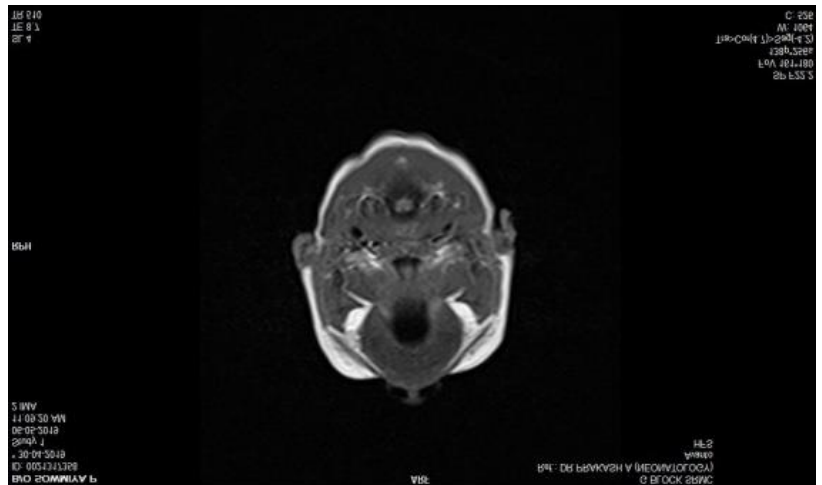

Figure 7. Input for power-law transformation based segmentation
Published By:

Blue Eyes Intelligence Engineering

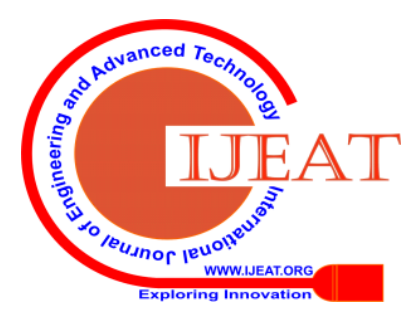




\section{Region of Interest Prediction using Segmentation}

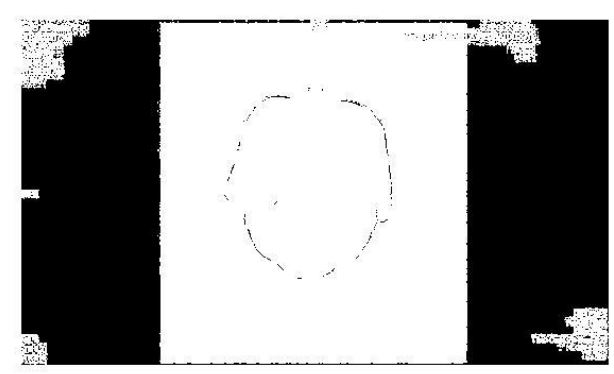

Figure 9. Output for power-law transformation based segmentation

The quality metrics for the above outputs are given in Table 4.

Table 4. Quality metrics for power-law transformation based segmentation

\begin{tabular}{|c|c|}
\hline MSE & 653.8644 \\
\hline SNR & 18.8525 \\
\hline PSNR & 20.2331 \\
\hline EPI & 0.7098 \\
\hline SSIM & 0.8990 \\
\hline
\end{tabular}

\subsection{Watershed Based Segmentation}

Watershed is a transformation defined on a grayscale image.There are two groups in watershed algorithms. Algorithms are used in the first group to create the flooding process.Watershed points are directly determined by the second group. Regional minima from the image is predicted and starting from minima, region growing is obtained. Consider the input given in figure 10.

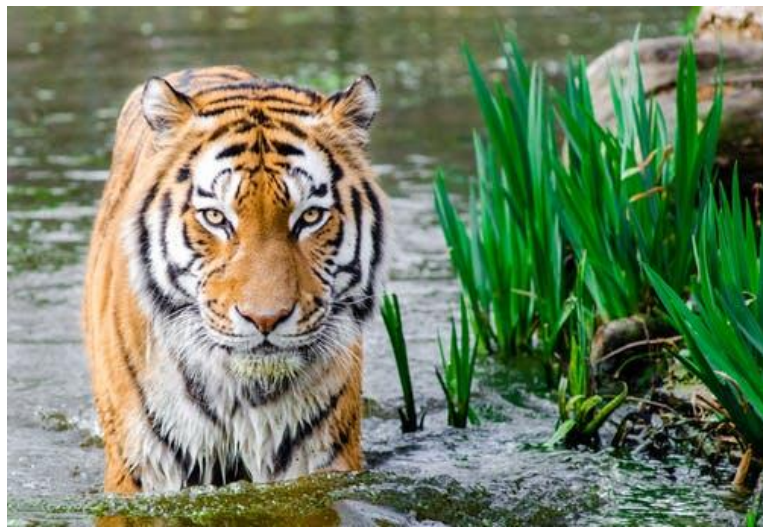

Figure 10. Input image for watershed based segmentation

The output of watershed transform is shown in figure 11 .

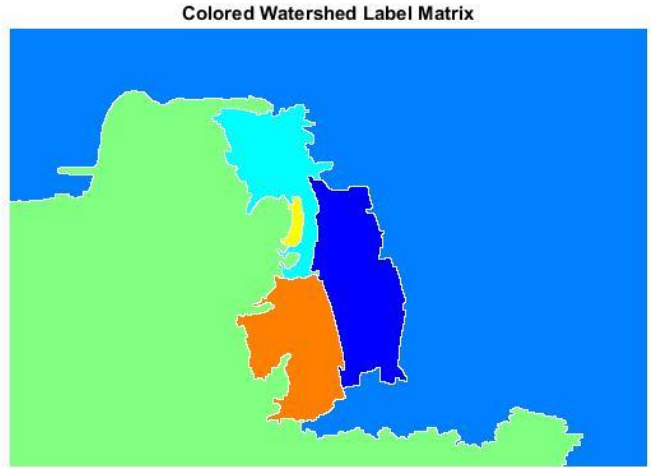

Figure 11. Output for watershed based segmentation
If the output is super-imposed on the original image the image is shown like figure 12 .

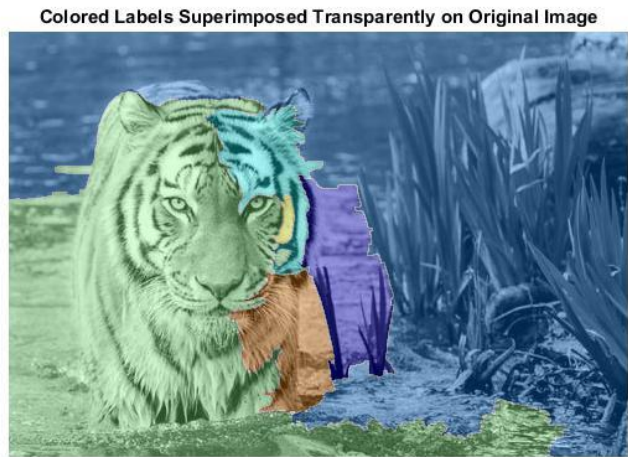

Figure 12. Watershed labels super-imposed on the original image

The quality metrics for the above outputs are given in Table 5 .

Table 5. Quality metrics for watershed transformation based segmentation

\begin{tabular}{|c|c|}
\hline MSE & 500.0728 \\
\hline SNR & 18.7667 \\
\hline PSNR & 21.2042 \\
\hline EPI & 0.6186 \\
\hline SSIM & 0.7805 \\
\hline
\end{tabular}

\section{CONCLUSION}

In this paper five techniques are implemented to various images, and their quality metrics MSE,SNR,PSNR,EPI and SSIM are calculated. Segmentation algorithm will be selected based on the values of Quality metrics and application. Among the quality metrics discussed in this paper, except MSE all other parameters value should be high to identify the quality of an image. Depends on the quality metrics, segmentation algorithm will be selected to predict the quality of an image

\section{REFERENCES}

1. Pillai, A.V.,Balakrishnan,A.A.,Simon,R.A.,Johnson, R.C.(2013), "Detection and localization of texts from natural scene images using scale space and morphological operations", International conference on Circuits, Power and Computing Technologies (ICCPCT), pp.880885.

2. L.M.Merlin Livingston and L.G.X.Agnel Livingston, "Processing of Images and Videos for Extracting Text Information from Clustered Features Using Graph Wavelet Transform”, Journal of Computational and Theoretical Nanoscience Vol. 16, 557-561, 2019.

3. Hase, H, Shinokawa, T., Yoneda, M. and Suen C. Y. (2001), "Character String Extraction from Color Documents", Pattern Recognition, Vol.4, No.7, pp.1349-1365.

4. Kim, K.I., Jung, K. and Kim, J.H. (2003), "Texture-based Approach for Text Detection in Image Using Support Vector Machine and Continuously Adaptive Mean Shift Algorithm", IEEE Transactions on Pattern Analysis and Machine Intelligence, Vol.25, No. 12, pp. 16311638.

5. Rafael G. Gonzalez and Richard E. Woods, Third edition Digital image processing. South Asia: Pearson, 2014.

6. S.Sridhar, Digital image processing. India: Oxford, 2011.

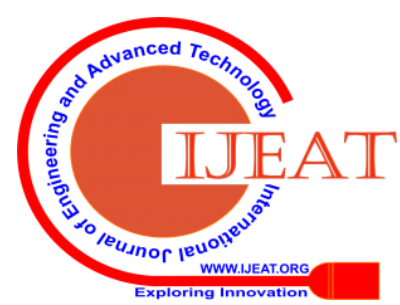


7. Clavelli, A. and Karatzas, D., 2009. Text Segmentation in Colour Posters from the Spanish Civil War Era. Proceedings of International Conference on Document Analysis and Recognition, pp.181-185, ISBN: 978-0-7695-3725-2,[DOI:10.1109/ICDAR.2009.32].

8. Ibtihal D. Mustafaand Mawia A. Hassan," A Comparison between Different Segmentation Techniques used in Medical Imaging",American Journal of Biomedical Engineering, 2016,pp. 5969.

9. Vaddi, R.S., Boggavarapu, L.N.P., Vankayalapati, H.D. and Anne, K.R.(2011), "Cellular neural network based pre-processing for localization of non standard licence plate", 3rd International Conference on Electronics Computer Technology (ICECT), Vol.1, pp. 407-411.

10. Kavita, RitikaSaroha, RajaniBala, and Ms.SunitaSiwach," Review paper on Overview of Image Processing and Image Segmentation", International Journal Of Research In Computer Applications And Robotics, Vol.1 Issue.7, October 2013, pp. 1-13.

\section{AUTHORS PROFILE}

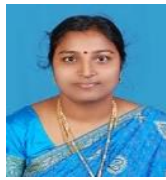

L. M. Merlin Livingston is currently working as professor in the department of Electronics and communication Engineering in Jeppiaar Institute of Technology,Chennai, Tamilnadu,India. Her areas of interest are Signal and Image Processing, Electronic ISTE,IEEE,ACM circuits and Embedded systems. She is a member of

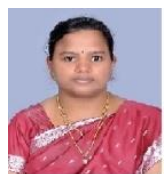

S. Mary Cynthia is currently working as a Assistant professor in the department of Electronics and communication Engineering in Jeppiaar Institute of Technology, Chennai, Tamilnadu, India. Her areas of interest are Signal and Image Processing, Antennas and Embedded systems. She is a member of ACM. 